\title{
New blind species and new records of Sinella from Nanjing, China (Collembola, Entomobryidae)
}

\author{
Heng Xu', Feng Zhang' \\ I College of Plant Protection, Nanjing Agricultural University, I Weigang, Nanjing 210095, P. R. China \\ Corresponding author: Feng Zhang (xtmtd.zf@gmail.com and fzhang@njau.edu.cn)
}

Academic editor: L. Deharveng | Received 25 January 2016 | Accepted 23 June 2016 | Published 11 July 2016

http://zoobank.org/27AFAE3B-EBDE-42D4-941A-79523A77BCFA

Citation: Xu H, Zhang F (2016) New blind species and new records of Sinella from Nanjing, China (Collembola, Entomobryidae). ZooKeys 604: 31-40. doi: 10.3897/zookeys.604.7902

\begin{abstract}
Two new blind species of Sinella are described from Nanjing, China. Sinella quinseta sp. n. from Purple Mountain possesses unique $5+5$ central macrochaetae on Abd. II, and can be distinguished from other species of the genus by the postlabial chaetae and the dorsal chaetotaxy. Sinella qixiaensis sp. n. from Qixia Mountain is characterized by the paddle-like S-chaetae of Ant. III organ and the smooth straight chaetae on the manubrium and base of dens; it differs from two closely related species by the smooth manubrial chaetae, the labial chaetae, the Ant. III organ, and the macrochaetae on Abd. II. Sinella fuyanensis Chen \& Christiansen and Sinella quinocula Chen \& Christiansen were also newly recorded from Nanjing.
\end{abstract}

\section{Keywords}

Springtail, eyeless, Sinella quinseta sp. n., Sinella qixiaensis sp. n.

\section{Introduction}

The genus Sinella has a worldwide distribution, and is particularly abundant in China. Deharveng (1990), Chen and Christiansen (1993) and Zhang et al. (2009, 2011) made main contributions to the modern taxonomy of this genus. To date, a total of 37 species, including 25 eyed (Ding and Zhang 2015) and 12 blind ones, have been reported from China. Among them, only four-eyed but no blind species were recorded 
from Nanjing, Jiangsu Province: Sinella curviseta Brook, 1882, Sinella triocula Chen \& Christiansen, 1993, Sinella affluens Chen \& Christiansen, 1993, and Sinella quinocula Chen \& Christiansen, 1993. In the present paper, two new blind species and two new records are reported from Nanjing.

\section{Materials and methods}

Specimens were cleared in Nesbitt's fluid, mounted under a coverslip in Marc André II solution, and studied using a Nikon E80i microscope. The labial chaetae terminology follows Gisin's system (1967). The dorsal and ventral chaetotaxy of head and the Ant. III organ are described after Chen and Christiansen (1993). Dorsal body chaetae are designated following Szeptycki (1979) and Zhang et al. (2011). The number of macrochaetae is given by half-tergite in the descriptions (left side of tergites drawn in figures). Tergal S-chaetotaxic formula follows Zhang and Deharveng (2015). All materials are deposited in the collections of the Department of Entomology, College of Plant Protection, Nanjing Agricultural University (NJAU), P. R. China.

\section{Abbreviations}

Th. thoracic segment;

Abd. abdominal segment;

Ant. antennal segment;

mac macrochaeta/ae;

mic microchaeta/ae;

ms S-microchaeta/ae;

sens ordinary tergal S-chaeta/ae.

\section{Taxonomy}

Sinella quinseta sp. $\mathbf{n}$.

http://zoobank.org/C83F07ED-4F4C-492D-A2B6-BBCCA5352450

Figs $1-18$

Material. Holotype: $q$ on slide, China, Jiangsu Province, Nanjing, Purple Mountain, Tomb of Liao Zhongkai and his wife He Xiangning, $32.056^{\circ} \mathrm{N}, 118.830^{\circ} \mathrm{E}$, altitude 38 m, 10 April 2009, Feng Zhang and Daoyuan YU leg. (\# C9581). Paratypes: $1 \hat{\delta}$ and $4 ㅇ ㅜ$ on slides and 5 juveniles in alcohol, same data as holotype.

Etymology. Named after the unique $5+5$ central mac on Abd. II in this new species.

Diagnosis. No eyes. Two internal sens of Ant. III organ expanded. Long smooth straight chaetae absent on antennae. Clypeal chaetae 7(5). Postlabial chaetae $\mathrm{X}, \mathrm{X}_{2}$ and 

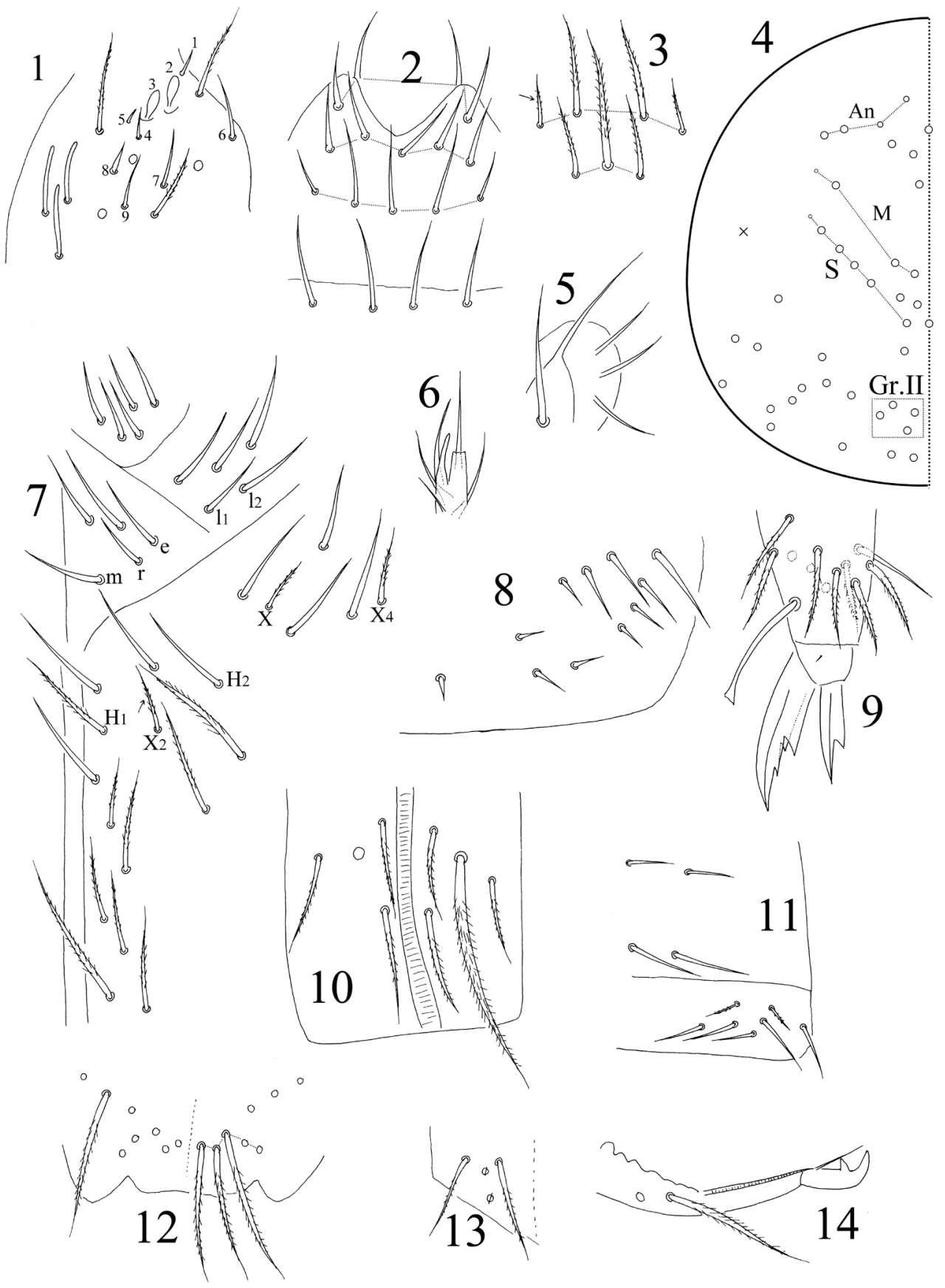

Figures I-I4. Sinella quinseta sp. n. I Ant. III organ $\mathbf{2}$ labrum $\mathbf{3}$ clypeal chaetae (arrow indicates that the chaeta may be absent) $\mathbf{4}$ dorsal cephalic chaetotaxy $\mathbf{5}$ maxillary outer lobe $\mathbf{6}$ lateral process of labial palp E $\mathbf{7}$ chaetae on the ventral side of head $\mathbf{8}$ trochanteral organ $\mathbf{9}$ hind claw $\mathbf{I 0}$ anterior face of ventral tube I I ventral face and lateral flap of ventral tube $\mathbf{I}$ distal part of anterior face of manubrium I 3 manubrial plaque 14 mucro. 
$\mathrm{X}_{4}$ ciliate. No "smooth" inner differentiated tibiotarsal chaetae. Tenent hairs clavate. Manubrium without smooth chaetae. Tergal ms as 1, 0|1, 0, 0, 0. Abd. II with 5+5 central mac. Abd. IV with 5+5 central and 5+5 lateral mac.

Description. Body length up to $1.17 \mathrm{~mm}$. Body pale in alcohol.

Antenna 1.69-1.80 times as long as cephalic diagonal. Antennal segments ratio as I $:$ II $:$ III $:$ IV $=1: 2.00-2.17: 1.82-1.90: 3.12-3.18$. Smooth spiny mic at base of antennae 3 dorsal, 3 ventral on Ant. I, 1 internal, 1 external and 1 ventral on Ant. II. Ant. II distally with 1 rod-like S-chaeta. Two internal sens of Ant. III organ expanded (Fig. 1). Long smooth straight chaetae absent on antennae.

Eyes absent. Prelabral and labral chaetae 4/ 5, 5, 4, all smooth; the three median chaetae of the row a longer than lateral ones (Fig. 2). Labral papillae absent. Clypeal chaetae 7(5), arranged in two rows; the inner two chaetae of the anterior row of four chaetae smooth in one specimen; most lateral two small chaetae absent in two specimens (Fig. 3). Dorsal cephalic chaetotaxy with four antennal (An), three median (M) and five sutural (S) mac; Gr. II with four mac (Fig. 4). Mandible teeth 4+5. Subapical chaeta of maxillary outer lobe thicker than apical one and subequal in length; three smooth sublobal hairs on maxillary outer lobe (Fig. 5). Lateral process of labial palp slightly thicker than normal chaetae, with tip beyond apex of labial papilla $\mathrm{E}$ (Fig. 6). Labial chaetae as mrel $1_{2}$, all smooth, $\mathrm{r} / \mathrm{m}=0.60-0.68$; chaetae $\mathrm{X}, \mathrm{X}_{2}$ and $\mathrm{X}_{4}$ ciliate; $\mathrm{X}_{2}$ often absent; chaeta $\mathrm{H}_{1}$ ciliate; $\mathrm{H}_{2}$ smooth in one specimen and ciliate in others. Cephalic groove with 9(8) chaetae, 2(3) smooth and others ciliate (Fig. 7).

Trochanteral organ with 9-13 smooth spiny chaetae; 7-9 in arms and 2-4 between them (Fig. 8). Inner differentiated tibiotarsal chaetae ciliate with ciliations not closely appressed to axis. Tibiotarsi distally with ten chaetae in a whorl. Unguis with three inner teeth; two paired teeth unequal, outer one large. Unguiculus with a large outer tooth. Tenent hairs clavate (Fig. 9). Abd. IV 2.44-3.32 times as long as Abd. III along dorsal midline. Ventral tube anteriorly with 4-5 ciliate chaetae; one of them much larger than others (Fig. 10); posteriorly with 4 smooth chaetae; each lateral flap with 5 smooth and 2 ciliate chaetae (Fig. 11). Manubrium without smooth chaetae. Manubrium anteriorly with $5+5$ ciliate chaetae in the most distal row (Fig. 12). Manubrial plaque with $2+2$ pseudopores and $2+2$ ciliate chaetae (Fig. 13). Distal smooth part of dens 1.34-1.85 times as long as mucro. Mucro bidentate with apical tooth longer than subapical one; basal spine long, nearly reaching tip of the apical tooth (Fig. 14).

Th. II with $3(\mathrm{~m} 1, \mathrm{~m} 2, \mathrm{~m} 2 \mathrm{i})$ medio-medial, three (m4, m4i, m4p) medio-lateral, 20-22 posterior mac, one ms and two sens; ms inner to sens. Th. III with 29-32 mac and two lateral sens; mac a6i absent (Fig. 15). Abd. I with six (a3, m2-4, m2i, m4p) mac, one ms and one sens; sens inner to ms. Abd. II with five (a2, a3, m3, m3e, m3ep) central, one (m5) lateral mac and two sens. Abd. III with two (a2, m3) central, three (am6, pm6, p6) lateral mac and two sens; ms absent (Fig. 16). Abd. IV with five central (I, M, B4, B5, A6), five lateral mac (E2-4, E2p, F1), and approximately 13 sens; as and ps shorter than others (Fig. 17). Abd. V with three sens; chaeta p5a absent (Fig. 18). 

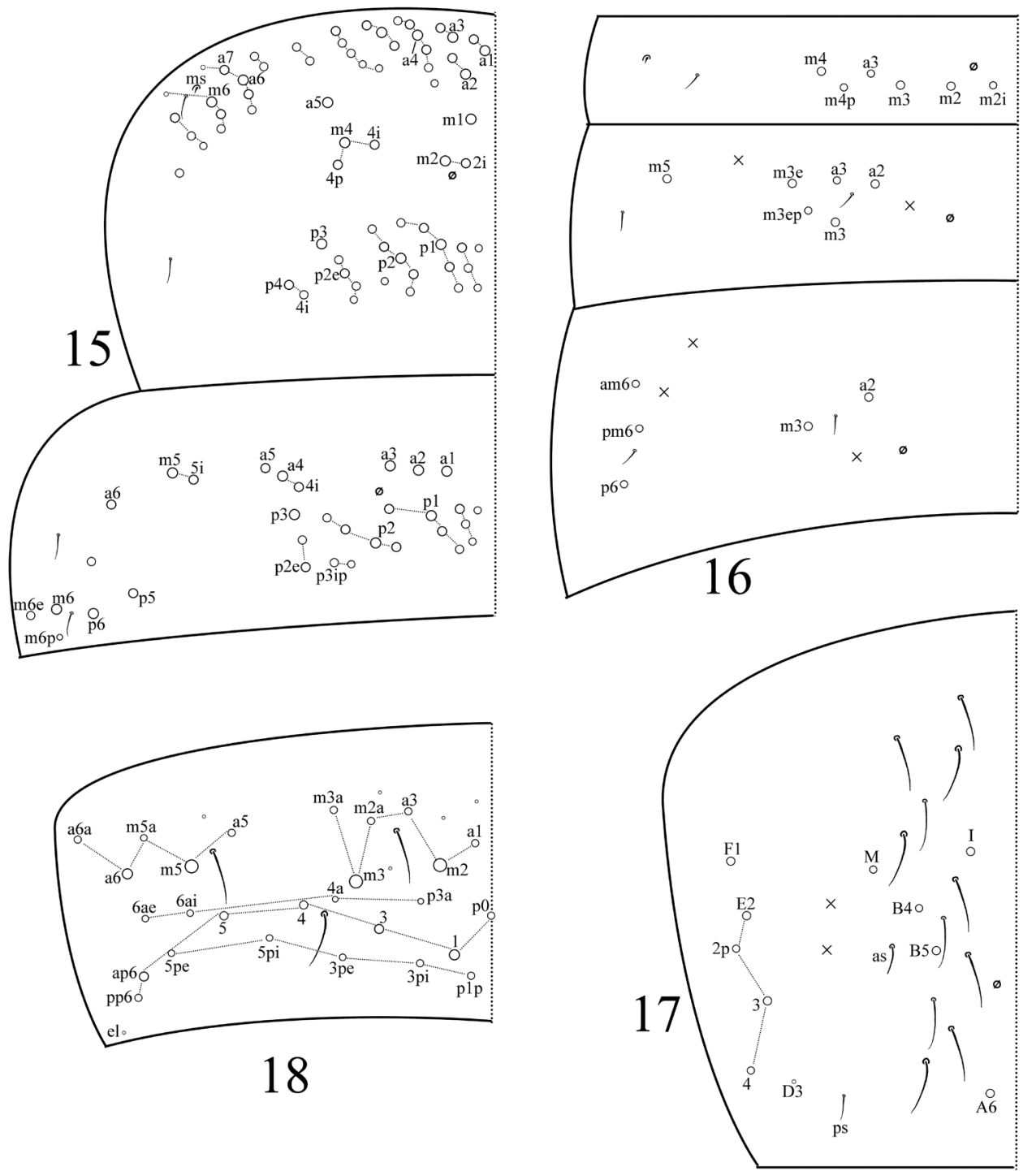

Figures 15-1 8. Tergal chaetotaxy in S. quinseta sp. n. 15 thorax 16 Abd. I-III 17 Abd. IV 18 Abd. V.

Ecology. In decomposing leaves along the roads.

Remarks. Sinella quinseta sp. n. is characterized by blindness, ciliate postlabial chaeta $\mathrm{H}_{1}$ and $5+5$ central mac on Abd. II. It is most similar to S. yunnanica Zhang \& Deharveng, 2011 in being blind, its claw structure, the lateral process of labial palp, mucro, and chaetotaxy of head, thorax and Abd. IV, but differs from it in the presence of expanded internal S-chaetae on Ant. III organ, ciliate $\mathrm{H}_{2}, \mathrm{X}, \mathrm{X}_{2}$ and $\mathrm{X}_{4}$ posterior to labium, $5+5$ central mac on Abd. II, and the ventral tube. 


\section{Sinella qixiaensis sp. $\mathbf{n}$.}

http://zoobank.org/DBFF3DC3-BC1D-4FC3-B5E3-5CB4E1D076E1

Figs 19-37

Material. Holotype: $\widehat{\gamma}$ on slide, China, Jiangsu Province, Nanjing, Qixia Mountain, $32.160^{\circ} \mathrm{N} 118.960^{\circ} \mathrm{E}$, altitude $114 \mathrm{~m}, 6$ December 2014, Daoyuan Yu and Chunyan Qin leg. (\#14NJQX4). Paratype: 490 on slides and 5 in alcohol, same data as holotype.

Etymology. Named after the type locality.

Diagnosis. No eyes. Two internal sens of Ant. III organ paddle-like. Long smooth straight chaetae present on antennae. Clypeal chaetae eight. Postlabial chaetae X and $\mathrm{X}_{2-4}$ ciliate. "Smooth" inner differentiated tibiotarsal chaetae present. Tenent hairs I and II pointed or clavate, and III always clavate. Manubrium with smooth chaetae. Tergal S-microchaetae as 1, 0|1, 0, 0, 0. Abd. II with 4(3) central mac on each side. Abd. IV with 7+7 central and 6+6 lateral mac.

Description. Body length up to $2.01 \mathrm{~mm}$. Body pale in alcohol.

Antenna 2.41-2.68 times as long as cephalic diagonal. Antennal segments ratio as I $:$ II $:$ III $:$ IV $=1: 1.66-1.96: 1.59-1.83: 2.44-3.00$. Smooth spiny mic at base of antennae three dorsal, three ventral on Ant. I, one internal, one external and two ventral on Ant. II. Ant. II distally with two rod-like sens. Two internal sens of Ant. III organ paddle-like (Fig. 19). Ant. IV with a knobbed subapical organ. Long smooth straight chaetae at least five ventral on Ant. I, at least 13 ventral on Ant. II, and one ventral on Ant. III.

Eyes absent. Prelabral and labral chaetae 4/ 5, 5, 4, all smooth; median three chaetae of the row a longer than lateral ones; labral intrusion not U-shaped (Fig. 20). Labral papillae absent. Clypeal chaetae eight in number, of which three are ciliated and small (Fig. 21). Dorsal cephalic chaetotaxy with four antennal (An), three median (M) and five sutural (S) mac; Gr. II with 5-6 mac (Fig. 22). Mandible teeth 4+5. Subapical chaeta of maxillary outer lobe larger than apical one; three smooth sublobal hairs on maxillary outer lobe. Lateral process of labial palp slightly thicker than normal chaetae, with tip beyond apex of labial papilla $\mathrm{E}$ (Fig. 23). Labial chaetae as $\mathrm{mrel}_{1} \mathrm{l}_{2}$, all smooth, $\mathrm{r} / \mathrm{m}=0.61-0.76$; chaetae $\mathrm{X}$ and $\mathrm{X}_{2-4}$ ciliate; chaeta $\mathrm{X}_{3}$ rarely absent. Cephalic groove with 8-9 chaetae, 4(5) of them smooth and others ciliate (Fig. 24).

Trochanteral organ with approximately 24 smooth spiny chaetae; $13-15$ in arms and 9-11 between them (Fig. 25). Partial inner differentiated tibiotarsal chaetae "smooth" with ciliations closely appressed to axis. Tibiotarsi distally with ten chaetae in a whorl. Unguis with three inner, one outer, and two lateral teeth; two paired inner teeth unequal, outer one large. Unguiculus with a large outer tooth. Tenent hairs I and II pointed or clavate, and III always clavate (Fig. 26). Abd. IV 3.13-4.67 as long as Abd. III along dorsal midline. Ventral tube anteriorly with $6+6$ ciliate chaetae, two of them much larger than others (Fig. 27); posteriorly with 12 smooth chaetae (Fig. 28); each lateral flap with eight smooth chaetae (Fig. 29). Male genital plate with seven pairs of projections and internally with one pair of small chaetae (Fig. 30). Manubrium dorsally with about $13+13$ smooth chaetae (Fig. 31); ventrally with 5+5 distal ciliate 

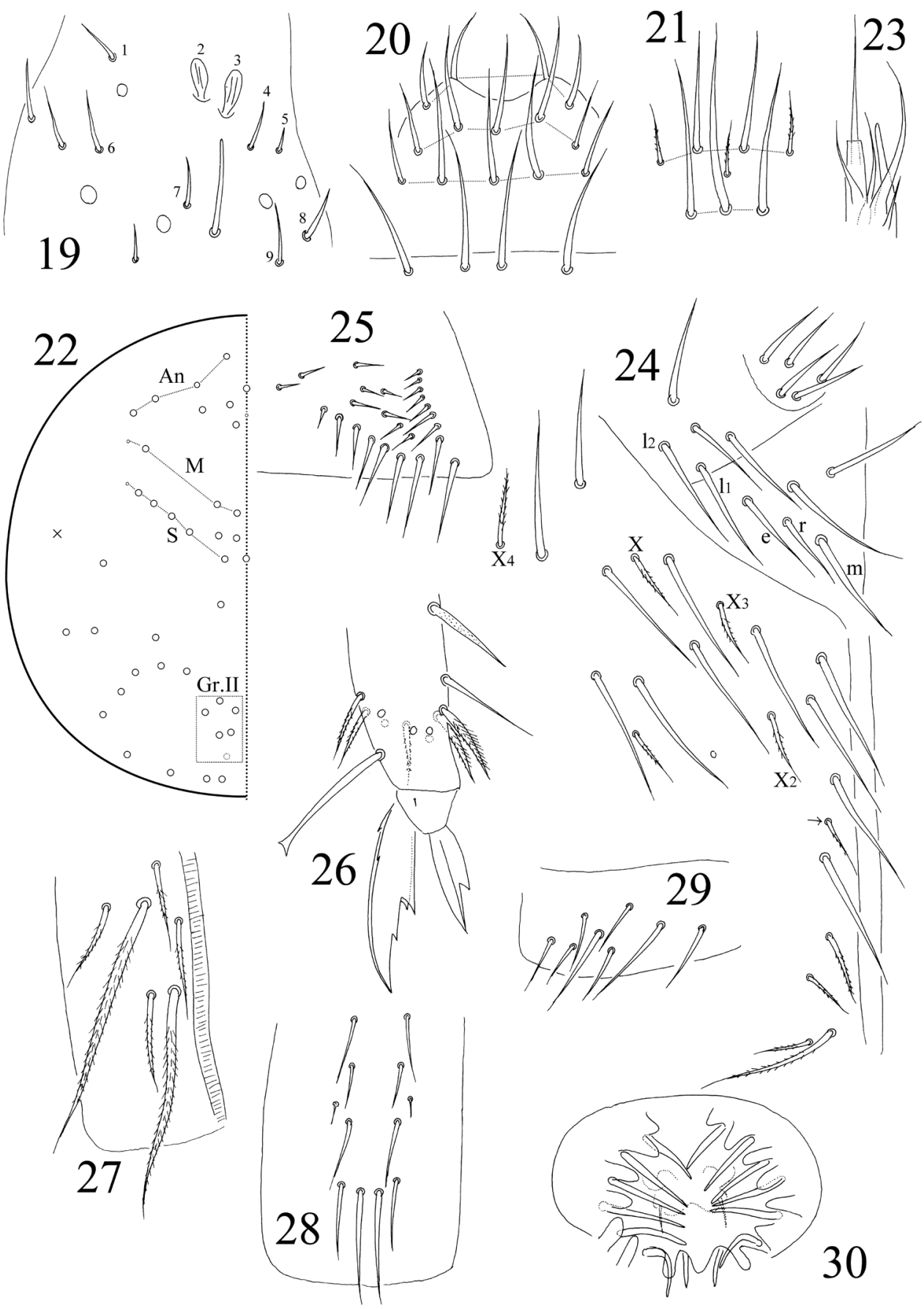

Figures 19-30. Sinella qixiaensis sp. n. 19 Ant. III organ 20 labrum 21 clypeal chaetae 22 dorsal cephalic chaetotaxy $\mathbf{2 3}$ labial palp $\mathbf{2 4}$ chaetae on the ventral side of head $\mathbf{2 5}$ trochanteral organ $\mathbf{2 6}$ hind claw 27-29 ventral tube $\mathbf{2 7}$ anterior face $\mathbf{2 8}$ posterior face $\mathbf{2 9}$ lateral flap $\mathbf{3 0}$ male genital plate. 


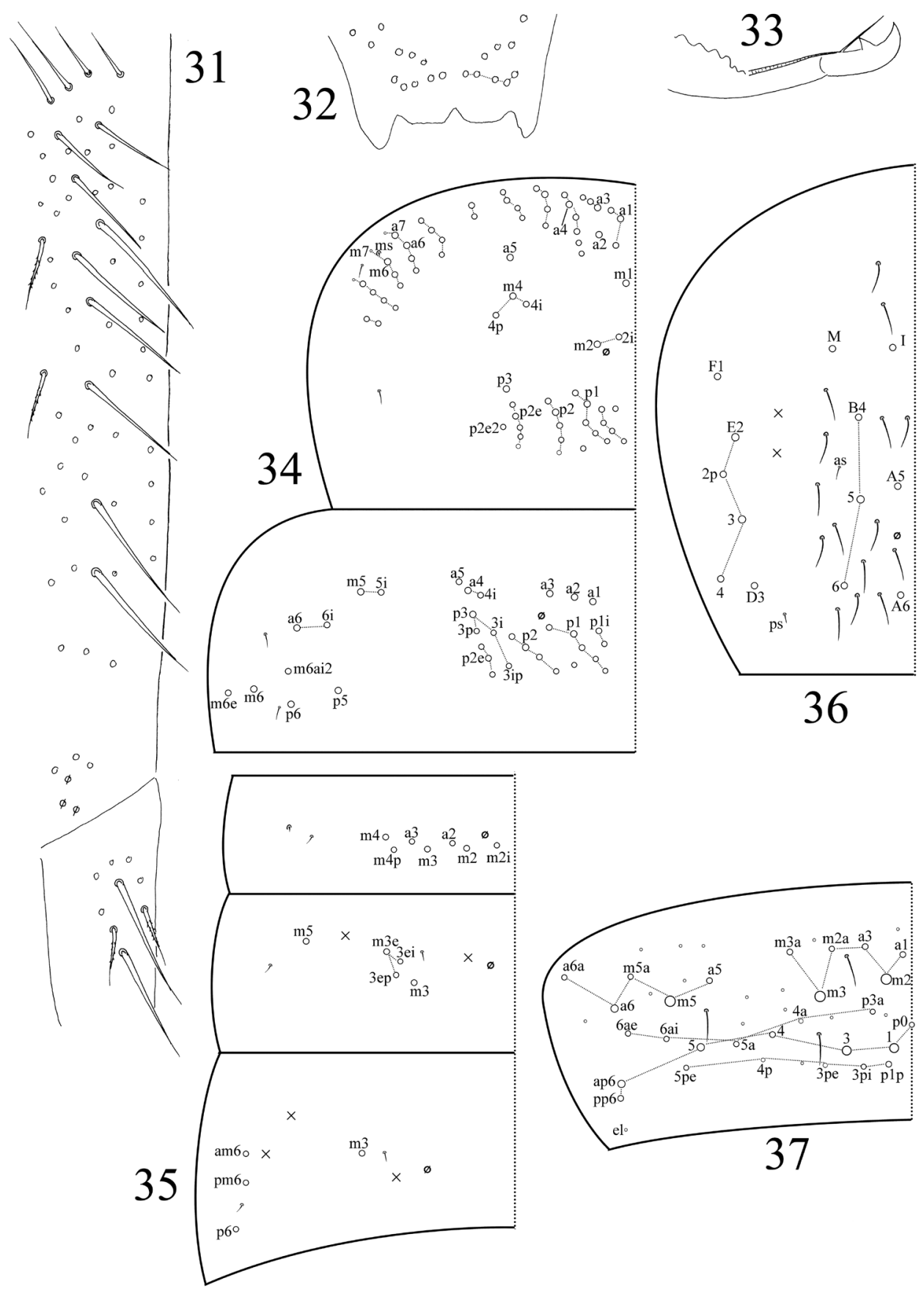

Figures $\mathbf{3}$ I-37. Sinella qixiaensis sp. n. $3 \mathbf{I}$ dorsal side of manubrium and base of dens $\mathbf{3 2}$ distal part of anterior face of manubrium 33 mucro 34-37 tergal chaetotaxy 34 thorax 35 Abd. I-III 36 Abd. IV 37 Abd. V. 
chaetae (Fig. 32). Manubrial plaque with 3+3 pseudopores and 3+3(2) ciliate chaetae. Base of dens with $2+2$ smooth chaetae (Fig. 31). Distal smooth part of dens 1.04-1.12 as long as mucro. Mucro bidentate with apical tooth larger; basal spine long, with tip nearly reaching apical tooth (Fig. 33).

Th. II with three $(\mathrm{m} 1, \mathrm{~m} 2, \mathrm{~m} 2 \mathrm{i})$ medio-medial, three $(\mathrm{m} 4, \mathrm{~m} 4 \mathrm{i}, \mathrm{m} 4 \mathrm{p})$ medio-lateral, 22-24 posterior mac, one $\mathrm{ms}$ and two sens; $\mathrm{ms}$ inner to sens. Th. III with 30-34 mac and two lateral sens; m5i, a6i, p5, p6, m6 and m6e as mac (Fig. 34). Abd. I with seven $(\mathrm{a} 2-3, \mathrm{~m} 2-4, \mathrm{~m} 2 \mathrm{i}, \mathrm{m} 4 \mathrm{p}) \mathrm{mac}$, one $\mathrm{ms}$ and one sens; sens inner to ms. Abd. II with 4(3) (m3, m3e, m3ep, m3ei) central, one (m5) lateral mac and two sens; mac m3ei only absent on one side of one specimen. Abd. III with one $(\mathrm{m} 3)$ central, three (am6, pm6, p6) lateral mac and two sens; ms absent (Fig. 35). Abd. IV with seven central (I, M, A5-6, B4-6), six lateral mac (D3, E2-4, E2p, F1), and at least 17 sens; sens as and ps short (Fig. 36). Abd. V with 3 sens (Fig. 37).

Ecology. In decomposing leaves along the roads.

Remarks. Sinella qixiaensis sp. n. is characterized by blindness, the paddle-like sens of Ant. III organ and abundant smooth chaetae on the manubrium. It is most similar to Sinella insolens Chen \& Christiansen, 1993 and Sinella sineocula Chen \& Christiansen, 1993. It differs from the former in the presence of smooth manubrial chaetae and the absence of labial chaeta $\mathrm{M}_{1 \mathrm{~s}}$, and also differs from $S$. sineocula in the presence of smooth manubrial chaetae, the paddle-like sens of Ant. III organ, and the presence of mac m3ei on Abd. II.

\section{Sinella fuyanensis Chen \& Christiansen, 1993}

Sinella (Sinella) fuyanensis Chen \& Christiansen, 1993: 27. Type locality: China (Jiangxi).

Material. + on slide and 4 in alcohol, China, Jiangsu Province, Nanjing, Lao Mountain, Long Cave, $32.051^{\circ} \mathrm{N}, 118.527^{\circ} \mathrm{E}$, altitude $112 \mathrm{~m}, 10$ April 2015, Daoyuan YU and Chunyan QIN leg. (\# 15NJLS).

Ecology. Known only from caves.

Distribution. China (Jiangxi, Jiangsu).

\section{Sinella quinocula Chen \& Christiansen, 1993}

Sinella (Sinella) quinocula Chen \& Christiansen, 1993: 24. Type locality: China (Anhui).

Material. $q$ on slide and 5 in alcohol, China, Jiangsu Province, Nanjing, Lao Mountain, Longxing temple, $32.051^{\circ} \mathrm{N}, 118.527^{\circ} \mathrm{E}$, altitude $112 \mathrm{~m}, 10$ April 2015, Daoyuan YU and Chunyan QIN leg. (\# 15NJLS).

Ecology. Under stones.

Distribution. China (Anhui, Jiangsu, Shaanxi). 


\section{Acknowledgments}

Thanks are given to Dr. Daoyuan YU and Chunyan QIN, who helped to collect animals. The present study was supported by the National Natural Sciences Foundation of China (41501056).

\section{References}

Brook G (1882) On a new genus of Collembola (Sinella) allied to Degeeria Nicolet. Journal of the Linnean Society of London Zoology 16: 541-545. doi: 10.1111/j.1096-3642.1882.tb02398.x

Chen J-X, Christiansen KA (1993) The genus Sinella with special reference to Sinella s. s. (Collembola: Entomobryidae) of China. Oriental Insects 27: 1-54. doi: 10.1080/00305316.1993.10432236

Deharveng L (1990) Fauna of Thai caves. II. New Entomobryoidea Collembola from Chiang Dao cave, Thailand. Occasional Papers of the Bernice P. Bishop Museum 30: 279-287. http://hbs.bishopmuseum.org/pubs-online/pdf/op30p279.pdf

Ding Y-H, Zhang F (2015) Contribution to the eyed Sinella from China: two new species and additional reports on nine known species (Collembola: Entomobryidae). Zootaxa 3973: 474-490. doi: 10.11646/zootaxa.3973.3.4

Folsom JW (1902) Collembola of the grave. Psyche 9: 363-367. doi: 10.1155/1902/42417

Gisin H (1967) Espèces nouvelles et lignées évolutives de Pseudosinella endogés (Collembola). Memórias e Estudos do Museu Zoológico da Universidade de Coimbra 301: 1-25.

Szeptycki A (1979) Morpho-systematic studies on Collembola. IV. Chaetotaxy of the Entomobryidae and its phylogenetical significance. Polska Akademia Nauk, Kraków, 219 pp.

Zhang F, Deharveng L (2015) Systematic revision of Entomobryidae (Collembola) by integrating molecular and new morphological evidence. Zoologica Scripta 44: 298-311. doi: $10.1111 /$ zsc. 12100

Zhang F, Deharveng L, Chen J-X (2009) New species and rediagnosis of Coecobrya (Collembola: Entomobryidae), with a key to the species of the genus. Journal of Natural History 43: 2597-2615. doi: 10.1080/00222930903243970

Zhang F, Yu D-Y, Xu G-L (2011) Transformational homology of the tergal setae during postembryonic development in the Sinella-Coecobrya group (Collembola: Entomobryidae). Contributions to Zoology 80: 213-230. http://www.ctoz.nl/cgi/t/text/get-pdf?c=ctz;idno$=8004 \mathrm{a} 01$ 